\title{
Strategie preventive dell'infezione da Citomegalovirus nel trapianto di rene
}

\author{
P. Carta, G. Rosso, N. Paudice, A. Larti, M. Zanazzi, L Moscatelli, L. Di Maria, \\ E. Bertoni
}

Unità Operativa Nefrologia dei Trapianti e Dialisi A.O.U. Careggi, Firenze

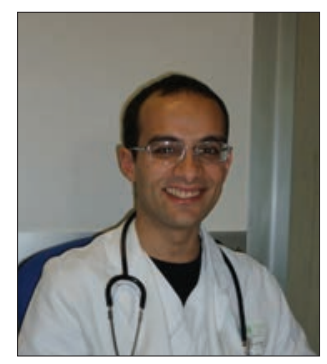

Paolo Carta

\section{Introduzione}

Il trapianto di rene rappresenta la migliore terapia sul lungo periodo per l'insufficienza renale cronica. L'uso della terapia immunosoppressiva è gravato da numerose complicanze tra cui le infezioni opportunistiche. Tra queste le infezioni da virus erpetici e in particolare quelle da citomegalovirus (CMV) rappresentano una complicanza frequente e un'importante causa di morbilità e mortalità per il paziente. La frequenza dell'infezione da CMV è giustificata dal fatto che circa l'80\% degli adulti sono sieropositivi per cui dopo il trapianto si può verificare una riattivazione dell'infezione stessa. L'altra via di contagio invece consiste nella trasmissione del virus in pazienti CMV "naïve" attraverso l'organo trapiantato o le trasfusioni ematiche. Nella popolazione generale l'infezione citomegalica si manifesta come una malattia simil influenzale o più spesso può decorrere in maniera asintomatica. Nel paziente immunodepresso la diagnosi non può prescindere dal riscontro della presenza del virus nel sangue del paziente mediante tecniche di PCR o di antigenemia PP53. Nel trapiantato renale quando la positività della CMV PCR non è associata a manifestazioni cliniche vale la definizione di infezione da CMV. Si parla invece di malattia da CMV quando si manifesta un qualsiasi sintomo o segno direttamente legato al virus. In particolare la malattia può assumere un quadro di malattia sistemica con interessamento di diversi organi o apparati tra cui i più tipicamente colpiti sono i polmoni, il tratto gastroenterico, l'apparato ematopoietico (in particolare può dare leucopenia) e la cute $(1,2)$. Inoltre in entrambi i casi di malattia e di infezione asintomatica si è dimostrato che il CMV è stato associato al verificarsi di effetti indiretti legati alla stimolazione del sistema immunitario come il rigetto acuto o cronico e le malattie linfoproliferative. In assenza di qualsiasi terapia preventiva l'infezione da CMV è stata riportata a seconda degli studi in approssimativamente il 30-75\% dei pazienti trapiantati e la malattia da CMV compresa tra l'8 e l'80\% a seconda del tipo di trapianto, del regime immunosoppressivo e soprattutto dello stato sierologico del donatore e del ricevente (presenza o meno di IgG specifiche) (3).

\section{Strategie terapeutiche}

Esistono due strategie attualmente in uso per la prevenzione della malattia da CMV: la profilassi e la terapia "preemptive". La prima consiste nella somministrazione di farmaci ad azione antivirale in pazienti senza infezione da CMV. Può essere iniziata nei primi 10 giorni dopo il trapianto e continuata generalmente per i primi 3 mesi dall'intervento ed è comunque indicata nei casi di riceventi $\mathrm{IgG}$ negativi per $\mathrm{CMV}$ che ricevono un rene da donatore IgG positivo per CMV (definiti R-/D+) o nei pazienti che hanno dovuto sostenere una terapia immunosoppressiva particolarmente pesante (per esempio, anticorpi antilinfocitari). La seconda invece prevede il controllo della CMV PCR ad intervalli regolari (generalmente settimanali) e l'inizio del trattamento in caso di riscontro di infezione asintomatica da CMV. Va comunque ricordato che il primo provvedimento da intraprendere, soprattutto nei casi a basso rischio immunologico e con bassa carica virale, è la riduzione della terapia immunosoppressiva generalmente mediante riduzione o sospensione dell'antimetabolita.

\section{Farmaci utilizzati}

I farmaci utili nella terapia dell'infezione del CMV sono l'Aciclovir, il Ganciclovir (disponibile in Italia solo per 
uso endovenoso), il Valganciclovir e il Valaciclovir per os. Nella pratica clinica in profilassi si preferisce utilizzare il Valganciclovir per via orale per il numero ridotto di compresse che è necessario assumere (è bene comunque ricordare che in Italia questo farmaco ha l'indicazione in scheda tecnica solamente per la terapia della retinite da CMV nei pazienti con AIDS anche se molte Regioni consentono l'utilizzo off-label con questa indicazione). Generalmente la durata della profilassi è di 3 mesi anche se un recente studio ha dimostrato una minore incidenza di malattia da CMV in pazienti $\mathrm{R}-/ \mathrm{D}+$ se la profilassi viene mantenuta per 200 giorni (4) .

La dose giornaliera dei farmaci antivirali utilizzati (sempre da correggere in base alla funzione renale del paziente) è la seguente:

Aciclovir per os $800 \mathrm{mg} \mathrm{x} 4$ /die

Ganciclovir ev: $5 \mathrm{mg} / \mathrm{kg} \times 2$ /die

Valaciclovir per os: 2 gr $\mathrm{x} 4$ /die

Valganciclovir per os: $900 \mathrm{mg} /$ die.

\section{Esperienza del nostro Centro}

Nella nostra esperienza abbiamo utilizzato la profilassi con Aciclovir per os a dosaggio aggiustato per la funzionalità renale fino al 2006. Dall'analisi di 100 pazienti trapiantati dal 2004 al 2006 in profilassi per 3-6 mesi, si è riscontrata una malattia citomegalica in 28 casi $(15$ casi di polmonite, 11 di enterite, 2 forme virali) (5). Successivamente abbiamo optato per la terapia preemptive con il Ganciclovir o con il Valganciclovir. In particolare di 200 pazienti $\mathrm{R}+/ \mathrm{D}+$ trapiantati dal dicembre $2007 \mathrm{al}$ dicembre 2010 si è evidenziata un'infezione da CMV in 28 casi (pari al 14\%) e una malattia da CMV in 20 casi (10\%), di cui 4 (14\% delle malattie riscontrate) nonostan- te la terapia preemptive. Nella nostra casistica dunque la strategia preemtpive con il Ganciclovir o il Valganciclovir pur risultando più efficace della precedente profilassi universale con l'Aciclovir, non è stata sufficiente a prevenire la malattia da CMV in 1 paziente su 10.

\section{Discussione}

Dai nostri dati e dalla revisione della letteratura più recente come anche dalle linee guida della Società Italiana di Nefrologia (6) emerge che la strategia di prevenzione della malattia citomegalica non è ancora perfettamente definita. Con sicurezza possiamo dire che è necessaria la profilassi con il Valganciclovir nei riceventi R-/D+. Per quanto riguarda i pazienti a basso rischio di malattia le evidenze disponibili a favore della terapia preemptive risultano meno forti rispetto alla profilassi. Ciò nonostante risulta problematico estendere la profilassi a tutti i pazienti sottoposti a trapianto renale anche considerando il fatto che, come già detto, il Valganciclovir non ha tale indicazione in scheda tecnica in Italia, e d'altra parte la terapia orale con Aciclovir o Valaciclovir è comunque molto impegnativa per il paziente. Ricordiamo inoltre il fatto che anche se il Valganciclovir ha un'efficacia paragonabile al Ganciclovir, il suo uso in profilassi si associa all'insorgenza di infezioni o malattie da CMV tardive che insorgono al termine della profilassi stessa e al verificarsi di fenomeni di resistenza al Ganciclovir. In diversi recenti studi comunque la strategia della profilassi universale (cioè anche per i pazienti CMV R+/D+) viene nuovamente presa in considerazione. Per Luan (7) la profilassi universale con Valganciclovir è risultata efficace clinicamente e vantaggiosa da un punto di vista economico rispetto alla strategia preemptive. A favore

\section{TABELLA I - PRINCIPALI LINEE GUIDA RIGUARDO LA PROFILASSI ANTI CMV}

\begin{tabular}{|l|l|l|}
\hline Linee guida & Raccomandazione & Cut off terapia preemptive \\
\hline Italiane 2007 (6) & $\begin{array}{l}\text { R-/D+: Profilassi con Valganciclovir per os } \\
\text { R+/D+: Preemtpive con il Ganciclovir ev } \\
\text { R-/D- : Da approfondire R-/D- }\end{array}$ & $\begin{array}{l}\text { Non indicazione su cut off della } \\
\text { PCRCMV }\end{array}$ \\
\hline Spagnole 2005 (9) & $\begin{array}{l}\text { R-D+: Profilassi con Valganciclovir - Valaciclovir per os o Ganciclovir ev } \\
\text { R+/D+: Preemptive Ganciclovir ev }\end{array}$ & $\begin{array}{l}\text { Cut off PCR CMV } 400 / 5000 \\
\text { copie/mL }\end{array}$ \\
\hline Europee $2000(12)$ & $\begin{array}{l}\text { Profilassi raccomandata nei R-/D+ e nei pazienti sottoposti a terapia con ATG / OKT3 } \\
\text { R+/D+: Preemptive }\end{array}$ & Non indicazioni su cut off \\
\hline Americane 2009 (13) & $\begin{array}{l}\text { Profilassi con Ganciclovir o Valganciclovir per os in R-/D+ e dopo trattamento } \\
\text { con anticorpi mono/policlonali } \\
\text { R+/D+: Preemptive }\end{array}$ & Non indicazioni su cut off \\
\hline
\end{tabular}


della profilassi universale ricordiamo poi il fatto che esistono buone evidenze che dimostrano che sia la malattia da CMV sia la viremia asintomatica sono fattori di rischio indipendente per lo sviluppo di rigetto acuto (8) e che i farmaci anti-virali usati contro il CMV sono efficaci anche nel ridurre l'incidenza di altre malattie virali causate da herpes simplex, varicella, Epstein Barr e HHV8 (l'agente eziologico del sarcoma di Kaposi).

Per quanto riguarda invece la strategia preemptive, un importante problema che emerge dalla revisione della letteratura è che non esiste un valore limite di copie $/ \mathrm{mL}$ di CMV PCR a cui iniziare il trattamento preemptive. In genere si raccomanda di basarsi su parametri del singolo laboratorio cui il Centro Trapianti fa riferimento in quanto le strategie diagnostiche attualmente in uso sono difficilmente standardizzabili. Sulla base della nostra esperienza un cut off di 100.000 copie $/ \mathrm{mL}$ è risultato troppo elevato ed è stato associato a sviluppo di malattia nel $14 \%$ dei pazienti sottoposti a terapia preemptive. A nostro giudizio sarebbe più opportuno iniziare la terapia a livelli di PCR CMV inferiore come suggerito da altre linee guida (per esempio, quelle spagnole suggeriscono di iniziare la terapia già a valori di PCR compresi tra 400 e 5000 copie $/ \mathrm{mL}$ ) (9). Al contrario, altri autori suggeriscono che la terapia preemptive può essere iniziata a livelli di 300.000 copie/ $\mathrm{mL}$ di PCR CMV senza rischio di sviluppare malattia sintomatica (10). Un secondo problema relativo alla strategia preemptive consiste poi nelle difficoltà organizzativogestionali e nei costi per effettuare i test della PCR CMV settimanalmente in tutti i pazienti per i primi mesi.

Esistono pochi trials che hanno confrontato le due strategie e generalmente hanno dato risultati non definitivi (11). Le varie linee guida (Tab. I) tendono a consigliare l'uso della profilassi solo nei pazienti ad alto rischio di malattia da CMV $(6,9,12,13)$. Comunque ciascun Centro deve bilanciare i rischi e i benefici dei due approcci tenendo presente la frequenza d'insorgenza di malattia da CMV nel proprio Centro, la possibilità di controllare la PCR CMV in tutti i pazienti, i costi della terapia antivirale e dei test per monitorizzare la PCR CMV, i tassi di insorgenza di malattia da CMV tardiva, la frequenza di altre infezioni virali opportunistiche, di rigetto, di perdita del graft e della mortalità del paziente (11).

\section{Indirizzo degli Autori:}

Paolo Carta, MD

Unità Operativa di Nefrologia dei Trapianti e Dialisi

A.O.U. Careggi Firenze

Viale Pieraccini 17

50100 Firenze

cartapa@aou-careggi.toscana.it

\section{Bibliografia}

1. Hartmann A, Sagedal S, Hjelmesaeth J. The natural course of Cytomegalovirus infection and disease in renal transplant patients. Transplantation 2006; 82 (Suppl): S15-7.

2. Fishman JA, Rubin RH. Infection in organ transplant recipients. N Engl J Med 1998; 338: 1741-51.

3. Pescovitz MD. Review of the CMV in renal transplantation. Saudi J Kidney Dis Transplant 2007; 18: 505-11.

4. Humar A, Lebranchu Y, Vicenti F, et al. The efficacy and safety of 200 days valganciclovir cytomegalovirus prophylaxis in high risk transplant recipients. Am J Transplant 2010; 10: 1228-37.

5. Paudice N, Mehmetaj A, Zanazzi M, et al. Preemptive therapy for the prevention of Cytomegalovirus Disease in renal transplant recipients: Our Preliminary Experience. Transplant Proc 2009; 41: 1204-6.

6. Cornella C, Torazza MC, Strippoli GFM, et al. Profilassi con farmaci antivirali e pre-emptive therapy per la prevenzione dell'infezione da citomegalovirus nel trapianto di rene: linee guida. G Ital Nefrol 2007; 24(Suppl 37): S165-78.

7. Luan FL, Kommareddi M, Ojo AO. Universal prophylaxis is cost effective in cytomegalovirus serology-positive kidney transplant patients. Transplantation 2011; 91: 237-44.

8. Reischig T. Cytomegalovirus-associated renal allograft rejection: new challenges for antiviral preventive strategies. Expert Rev Anti Infect Ther 2010; 8: 903-10.

9. Torre-Cisnero J, Fortun J, Aguada JM, et al. Recomendaciones GESITRA (grupo de estudio de la infecciòn en el transplante) - SEIMC (Sociedad española de microbiologia clinica y infecciòn en el transplante) sobre prevenciòn $\mathrm{y}$ tratamiento de la infecciòn por citomegalovirus en paziente transplantados. Enferm Infec Microbiol Clin 2005; 23: 424-37.

10. Gerna G, Baldanti F, Torsellini M, et al. Evaluation of citomegalovirus DNAaemia versus pp65-antigenaemia cutoff for guiding preemptive therapy in transplant recipients: a randomized study. Antivir Ther 2007; 12: 63-72.

11. Kotton C. Management of cytomegalovirus infection in solid organ transplantation. Nat. Rev. Nephrol 2010; 6: 711-21.

12. Berthoux F, Abramowics D, Bradley B. European best practice guidelines for renal transplantation (part 1) Nephrol. Dial. Transplant 2000; 15 (Suppl 7): S71-4.

13. Kidney Disease: Improving Global Outcomes (KDIGO) Transplant Work Group. KDIGO clinical practice guideline for the care of kidney transplant recipients. Am J Transplant 2009; 9 (Suppl 3): S44-S58. 